\title{
Conocimiento ancestral en la curación de la próstata a base de isaño (Tropaeolum tuberosum Ruiz y Pavón)
}

\author{
Ancestral knowledge in healing the prostate based on isaño \\ (Tropaeolum tuberosum Ruiz y Pavón)
}

\author{
Emilio Flores Mamani ${ }^{1 *}$, Jorge Apaza Ticona ${ }^{1}$, Wilber César Calsina Ponce ${ }^{1}$, \\ Germán Quille Calizaya ${ }^{1}$, Félix Huanca Rojas ${ }^{1}$, Alejandro Coloma Paxi ${ }^{1}$, \\ Juan Inquilla Mamani ${ }^{1}$, Percy Huata Panca ${ }^{1}$, Arturo Zayra Churata ${ }^{1}$
}

\section{RESUMEN}

\begin{abstract}
Los conocimientos ancestrales respecto a la medicina tradicional son importantes debido a que tuvieron presencia en la lucha contra diversas enfermedades, cuando no había hospitales ni profesionales de salud. Sin embargo, en la actualidad están siendo subestimados y dejados de lado por la ciencia. Muchos pobladores altoandinos y de otros lugares sufren de la inflamación de la próstata, que antes se curaba con el isaño. El objetivo de este artículo es sistematizar los saberes de la práctica del tratamiento de la prostatitis a través del isaño cultivado (para consumo) y pajarillu (isaño silvestre) en las comunidades campesinas de las provincias de Yunguyo, Chucuito, El Collao y Puno de la región de Puno - Perú. El enfoque de esta investigación es cualitativo, método etnográfico y fenomenológico. La población de estudio fueron adultos mayores que saben sobre la prevención y curación de los problemas de próstata con isaño. Se utilizó la técnica de la entrevista semiestructurada para obtener la información de campo. El resultado sugiere que el isaño silvestre o pajarillu es el tubérculo que cumple el papel curativo de la próstata y no el isaño cultivado, que solo cumple el papel de prevención. Se concluyó que muchas personas con enfermedades de la próstata fueron sanadas por curanderos a base de isaño silvestre.
\end{abstract}

Palabras clave: conocimiento ancestral, isaño de consumo, isaño pajarillu, curación de la próstata.

\begin{abstract}
Ancestral knowledge of traditional medicine is important because they were present in the fight against many different diseases when there were no hospitals or health care professionals, nowadays this is being underestimated and overlooked by science; many High Andean people and from other places suffer from prostate inflammation, which used to be cured with isaño.The objective of the article is to systematize the knowledge of the practice of the treatment of the prostate through the cultivated isaño (for consumption) and pajarillu (wild isaño) in the rural communities of the provinces of Yunguyo, Chucuito, El Collao and Puno of the region from Puno - Peru. The research approach is qualitative, ethnographic and phenomenological, the population of study were elderly people who know about prevention and healing of the prostate with isaño. The technique of semi-structured interview was used to obtain information from the field. The result reveals that the wild isaño or pajarillu is the tuber that fulfills the healing role of the prostate of people suffering from the disease and is not the cultivated isaño, which only fulfills the role of prevention. It is concluded that many people with prostate disease were healed by healers based on the pajarillu isaño.
\end{abstract}

Keywords: ancestral knowledge, consumption isaño, pajarillu isaño, healing of the prostate.

\section{Introducción}

La medicina tradicional es como una escala de saberes ancestrales, habilidades, creencias, que forman un sistema que permite prevenir y tratar problemas de salud. Se trata de la aplicabilidad de las plantas medicinales para curar determinadas enfermedades (Velásquez et al., 2013). Sin embargo, esa sapiencia de miles de años está desapareciendo paulatinamente junto con los pobladores antiguos que usaban las plantas naturales. Desde las ciencias sociales se enfocó la historia de la medicina tradicional, considerando los factores culturales, la enfermedad, el sufrimiento $\mathrm{y}$ todo cuanto atañe al hombre y su salud, abarcando las dimensiones biológica, cultural, psicológica y espiritual (Perdiguero y Comelles, 2000).

El isaño o mashua (Tropaeolum tuberosum Ruiz y Pavón) es una planta que produce tubérculos en

1 Universidad Nacional del Altiplano de Puno. Puno, Perú.

* Autor para correspondencia: eflo207@hotmail.com 
lugares altoandinos de Sudamérica. Es conocido también como añu en Bolivia y cubio en Colombia (Morillo et al., 2016; Guevara-Freire, et al., 2018). Geográficamente se extiende desde Colombia hasta Bolivia entre 1500-4200 msnm, y su hábitat son los lugares frígidos (Aruquipa et al., 2017). El color de la piel del isaño varía del blanco marfil al púrpura morado muy oscuro, pasando por el amarillo, naranja y púrpura morado en distintas tonalidades y tamaños (Inostroza et al., 2015). Esas características permiten suponer una gran cantidad de micro y macronutrientes además de los compuestos bioactivos (Benítez et al., 2016; Malice et al., 2010). Existen dos especies: la ssp. tuberosum que es la domesticada, cultivada y para el consumo; y la ssp. silvestre Sparre, muy poco conocida (Bulacio y Ayarde, 2012).

Los pobladores altoandinos consumen el isaño para balancear su alimentación y aprovechar sus atributos nutricionales, antiafrodisiacos y medicinales. Dentro de sus usos medicinales se emplea como tratamiento en litiasis renal, dolencias genitourinarias y anemia. También se le atribuyen propiedades antimicrobianas (Aire - Artezano et al., 2013). Existen estudios in vitro (16-19) que destacan la capacidad antioxidante de este tubérculo debido a su alta concentración en compuestos fenólicos, antocianinas y carotenoides (Cruz et al., 2017). En general, los tubérculos de mashua mostraron la mayor capacidad antioxidante y el contenido de fenólicos, antocianinas y carotenoides en comparación con la papa, oca y el ulluco (Campos et al., 2006).

En los tiempos actuales hay mayor interés en los antioxidantes naturales debido a sus efectos biológicos beneficiosos para el ser humano, como antibacterianos, antivirales, antialérgicos, antitrombóticos, y porque son vinculados a una menor incidencia de enfermedades cardiovasculares y ciertos tipos de cáncer (Espinoza y Monteghirfo, 1999; Salluca et al., 2008; Vásquez et al., 2012). Sin embargo, se considera que las dosis más bajas de mashua reducen el número y la calidad del esperma (motilidad y morfología), y el efecto de la administración de dosis más altas puede ser reversible 24 días después de la finalización del tratamiento (Leiva-Revilla et al., 2012). Como indican los autores, el isaño tiene atributos nutracéuticos para el hombre, y por eso, algunas poblaciones rurales del altiplano lo utilizan para curar ciertos problemas de salud.
De acuerdo con Valcárcel-Yamani et al. (2013), la mashua contiene la más alta cantidad de vitamina C (77,37 mg en $100 \mathrm{~g}$ de materia fresca) de todos los tubérculos. Además su alto contenido de provitamina A lo convierte en uno de los tubérculos con más carotenos, con un nivel promedio de 73,56 RE-/-100 g de materia fresca. Uno de los principales componentes del isaño es el almidón (Velásquez-Barreto y Velezmoro, 2018). Asimismo tiene una humedad de $88,7 \%$; ceniza, $4,81 \%$; proteínas, 9,17\%; fibra, 5,86\%; fósforo, $0,32 \%$; potasio, $1,99 \%$ y energía, $440 \mathrm{Kcl} / 100 \mathrm{~g}$ (ValleParra et al., 2018). Estos y otros componentes del tubérculo posibilitan la curación de enfermedades de las poblaciones altoandinas.

Muchos pobladores altoandinos o de lugares frígidos rurales y urbanos sufren el problema de la inflamación de la próstata, por lo que se ven afectados psicológicamente en su vida cotidiana, al no poder realizar sus actividades con normalidad. En este contexto, la utilización del isaño cultivado y el silvestre por parte de los habitantes altoandinos para tratar las enfermedades de la próstata sigue vigente en algunas comunidades campesinas del departamento de Puno-Perú, que le atribuyen efectividad en la sanación de la prostatitis. De ser así, podría ayudar a muchos hombres para solucionar ese problema. En este sentido, el objetivo del estudio fue sistematizar los conocimientos ancestrales de la práctica del tratamiento de la próstata a través del consumo de isaño cultivado y el silvestrepajarillu- en las comunidades campesinas de las provincias de Yunguyo, Chucuito, El Collao y Puno de la región de Puno-Perú.

\section{Metodología}

\section{Lugares de estudio}

Casi en todas las provincias de la región de Puno se cultiva el isaño de consumo, excepto en Sandia debido a la temperatura media mayor que en otras localidades. De la misma forma, el pajarillu crece libremente en el campo altoandino en lugares alejados y en algunos casos es ornamental.

El centro poblado de Sanquira, lugar de estudio, está ubicado a 11 kilómetros al sur de la provincia de Yunguyo y es una zona agrícola. Allí se cultiva el isaño en distintas variedades y también existe el pajarillu que crece libremente alrededor de los pedregales. Otro sitio estudiado 
es el centro poblado de Pallalla, que se encuentra a 34 kilómetros al sur de la ciudad de Puno, en jurisdicción del distrito de Platería, y cuya actividad económica es principalmente la agroganadería. Asimismo, el centro poblado de Molino, ubicado en la carretera panamericana, a $10,5 \mathrm{~km}$ al sur de la ciudad de Juli, un excelente lugar para el cultivo de isaño. La población se dedica a la agricultura y a la ganadería como actividad complementaria. También se visitó la comunidad de Maquercota, que se encuentra a 10 kilómetros de la capital de la provincia de El Collao y su jurisdicción distrital es Pilcuyo. Todos los lugares incluidos en el estudio están ubicados en los alrededores del lago Titicaca.

\section{Enfoque de investigación}

El estudio se desarrolló dentro del enfoque de investigación cualitativa, método etnográfico y fenomenológico, el cual tiene como premisa la necesidad de comprensión del sentido de la acción social en el contexto del mundo de la vida y desde la perspectiva de los participantes (Vasilachis, 1992). Para la recolección de la información se utilizó la técnica de la entrevista semiestructurada a los pobladores que tuvieran experiencia de productor, consumidor de isaño y cultura ancestral en la curación de enfermedades. Es decir, los participantes fueron seleccionados de manera intencionada en función de su vinculación con el tema y los objetivos de la investigación. Son considerados informantes claves al tener la posibilidad, disposición e interés de referirse a su experiencia y dar a conocer los significados de los conceptos que guían la investigación.

Se entrevistó a 8 personas mayores de 50 años, 2 informantes por cada zona adscrita al estudio, que cultivan y practican la curación con isaño como medicina natural, localizados en diferentes provincias del departamento de Puno en zonas con mayor producción de este tubérculo. Se formularon diez (10) preguntas semiestructuradas o generales respecto a los saberes de las propiedades nutracéuticas del isaño, así como a la aplicación en el proceso de curación de prostatitis. Una vez que se obtuvieron las informaciones de los entrevistados se formularon más preguntas diferidas para redondear la idea o el significado.

En cada sitio de estudio se hicieron visitas previas a las entrevistas a fin de identificar a los informantes claves. Para ello fue necesario contratar guías que ayudaran a llegar a los lugares donde se encuentran los informantes y hacer el contacto con ellos. Allí se localizó a los informantes claves, los llamados curanderos de la próstata (prostatitis), para conocer su versión respecto al tratamiento de sus pacientes a base de isaño y pajarillu.

El procesamiento general de análisis de datos cualitativos fue el modelo que proponen Rodríguez et al. (1999), donde se efectúan tres acciones fundamentales: reducción de datos, disposición y transformación de datos, y obtención y verificación de conclusiones. De esta forma los datos fueron analizados y luego interpretados según las categorías sobre los saberes y la práctica de curación con isaño de los problemas de próstata.

\section{Análisis de datos}

En el trabajo de campo realizado entre los meses de diciembre 2019, enero y febrero de 2020, se recolectó valiosa información sobre la sapiencia ancestral referente a las bondades del isaño y pajarillu, que son utilizados por los pobladores rurales de la región de Puno para su alimentación y la curación de la próstata.

\section{Cultivo de isaño comestible e isaño silvestre - pajarillu}

El isaño es cultivado en una altura por encima de los 3800 msnm en la región de Puno, donde el clima es frígido y seco, hábitat apropiado del isaño comestible y el silvestre. Respecto al cultivo en las zonas de estudio se tiene el testimonio del habitante del centro poblado de Ticona Cusullaca del distrito de Pilcuyo, provincia de El Collao: " $L a$ siembra de Isaño se hace en suelo negro arenosas y siempre tenemos que realizar en los meses de agosto a octubre y la cosecha la efectuamos en los meses de abril y mayo, también depende de la campaña agrícola, si la campaña es con bastante lluvia los tubérculos en general producen bastante" $(\mathrm{E}=1)$.

El otro entrevistado del centro poblado de Sanquira - Yunguyo, que también es productor, señala que se cultivan y cosechan en la misma época que menciona el anterior informante. En cuanto al isaño silvestre o pajarillu dijo: "El pajarillu es silvestre, puede crecer libremente en el campo, pero, en los últimos años algunos pobladores tienen como planta de adorno en su 
casa, sin saber que sus raíces o los tubérculos tienen un importante valor curativo" $(\mathrm{E}=2)$.

Se presentan las imágenes de las plantas de isaño tanto de consumo como el pajarillu (Figura 1 y Figura 2).

Respecto a la tierra de cultivo, el informante del centro poblado de Molino de la provincia de Chucuito - Juli refiere que: "el isaño se siembra en toda la comunidad, la semilla es lo que les deja cada año, es original de este lugar, se guarda una parte para el consumo y la otra para la semilla $(E=3)$. Sabe curar el malestar de la próstata. Sin

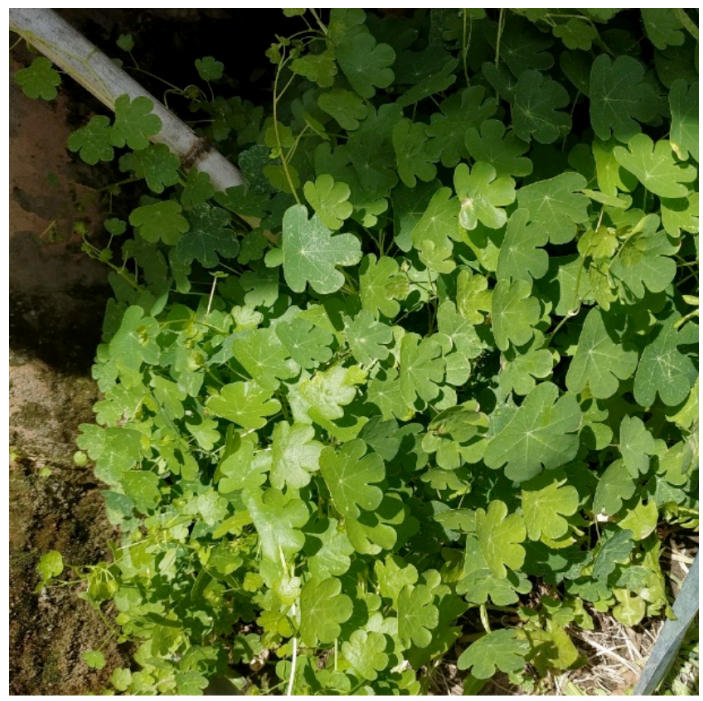

Figura 1. Planta de isaño silvestre o pajarillu.

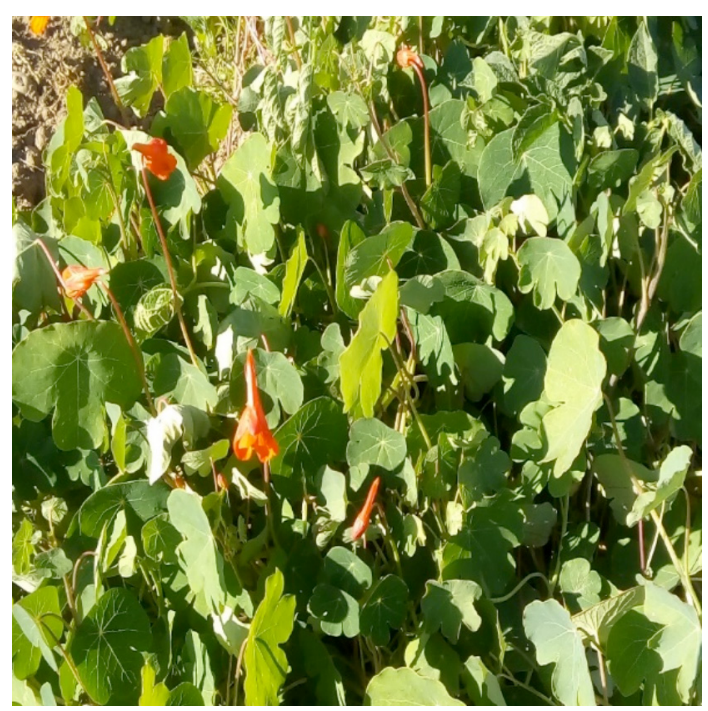

Figura 2. Planta de isaño de consumo. embargo, el informante de la comunidad de Pallalla del distrito de Platería - Puno señala que "la tierra negra es el apropiado para cultivar isaño; antes la producción era buena, cada plantón de isaño producía entre 80 a 100 unidades de gran tamaño, hoy en día solo produce entre 20 a 30 unidades en los mejores casos" $(\mathrm{E}=4)$.

Esto permite suponer que el isaño no se puede cultivar en otras regiones con características diferentes a las de la zona altoandina, debido a las particularidades naturales de la planta que tiene como hábitat la altura.

\section{Variedades de isaño de consumo y silvestre - pajarillu}

Algunos entrevistados señalan que el isaño cultivado en esta región presenta diversas características según su color y textura. Entre las variedades se encuentran amarillo de ojos violeta, amarillo zapallo y negro. "Hay varias clases de isaño de consumo diferenciado por su color blancoamarillo, amarillos medios verdes o alimonados y negros que son utilizados para el consumo humano. Asimismo, el isaño pajarillu se clasifica en color negro, blanco, rosado y amarillo, todos estos de contextura delgada y tamaño largo" (E - 2). En Molino, además de las mencionadas clases, según el informante, "hay isaño amarillo, blanco humo, ojos negros y color negro" $(E=3)$. De esta forma el isaño es cultivado en variedades para el consumo de los pobladores locales, donde se destaca la particularidad de cada lugar de siembra.

Los testimonios de los entrevistados se pueden corroborar con las imágenes obtenidas desde los sitios de estudio (Figuras 3 y 4).

\section{Uso del isaño para la alimentación}

En las comunidades campesinas de la región altoandina el isaño es parte de su alimentación. En cada campaña agrícola siembran, cosechan y almacenan para abastecerse durante el año. Generalmente es consumido sancochado, previa exposición al sol para quitarle la acidez y que se vuelva dulce. Según Carpio et al., 2016, además de sancochado, puede tener otras presentaciones como mermelada, macerado, licor terapéutico y extracto. Sobre este tema el entrevistado expresa: "En mi zona todos comemos el isaño; para sancochado tenemos que solear y para la thayacha 


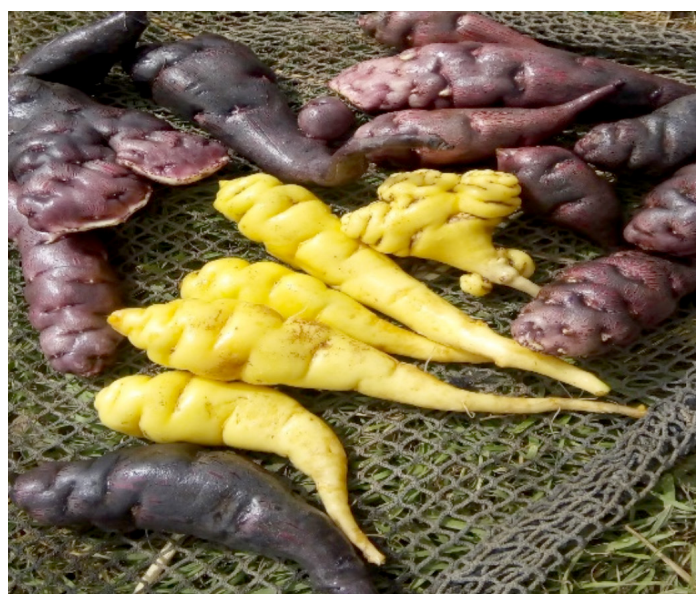

Figura 3. Isaño de consumo.

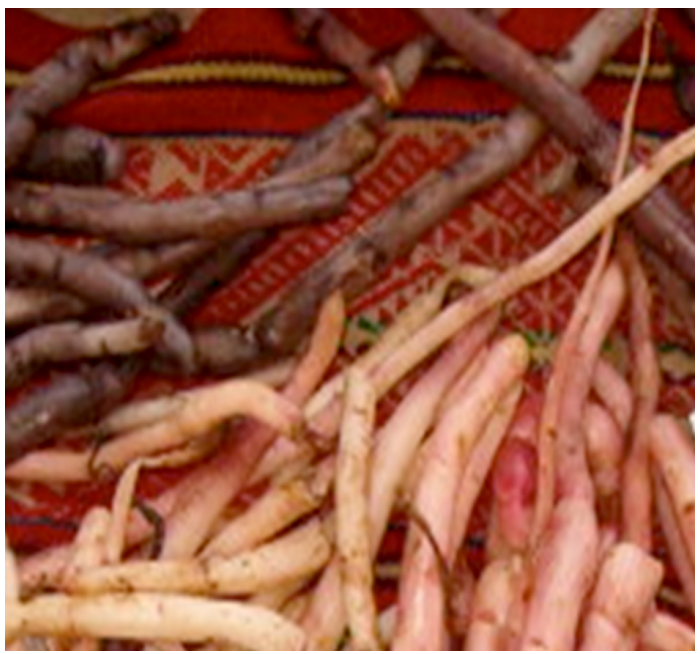

Figura 4. Isaño silvestre o pajarillo.

(congelado) también tiene que ser soleado, para que sea dulce, la thayacha se consume en el mes de junio (época de helada), también se consume en la sopa el isaño. Bueno, no se puede hacer el chuño de isaño (deshidratación). Nosotros la conocemos al isaño como engreídas porque se necesita mucho cuidado en almacenamiento, se malogra rápido" $(E=5)$.

En la provincia de Chucuito-Juli el isaño es consumido en diferentes presentaciones, como explica el informante: "El amarillo, se cocina en vez de zapallo, en el caldo cocinan, blanco humo, se sancocha, ojos negros, también se sancocha, negros, se sancocha, después de sancochado se hace congelar con la helada, (thayacha)... Hay que solearlo igual que la oca y luego recién se cocina, y tiene más rico sabor. Hay que solearlo uno o tres días y se vuelve dulce. No se come crudo porque es amargo y veneno... jajaja... Puré de isaño, se hace cocer y se muele con leche y azúcar, se hace hervir un poquito, eso se llama puré" $(E=3)$. Deben ser consumidos de vez en cuando.

En Pallalla, "primero se hace solear, se consume en sopa de isaño, sancochado y thayacha... yo como dos o tres veces a la semana, no sufro de la próstata" $(\mathrm{E}=4)$.

De esta forma concluimos que el isaño es un alimento habitual en las comunidades campesinas y se consume sancochado. Todavía no se ha ofrecido en el mercado en otras presentaciones que puedan motivar su consumo para prevenir los problemas de próstata.

\section{Saberes de la población sobre las bondades del isaño}

En su cultura medicinal la población rural de la región andina considera a las plantas de su entorno como elementos bioactivos para curar ciertas enfermedades, conocimiento que heredaron de sus antepasados desde hace miles de años. Una de esas plantas es el isaño que produce los tubérculos usados como medicina.

Las entrevistas demuestran que también las mujeres usan el isaño para los problemas renales, tal como explica la informante: - "Después de pasar muchos dolores, se resquebrajó mi salud, por eso ido al hospital de Puno. El médico me diagnosticó cálculos en los riñones, está avanzado me dijo y me recetó unos medicamentos para los dolores, creo que se venía lo peor para mí" $(E=6)$. - La mujer volvió a su pueblo hecha un "paño de lágrimas", pensando en que se iba a morir. "Lloraba todos los días en mi casa hasta que se había enterado la curandera de mi pueblo que me regañó primero y luego me recetó tomar durante 20 días agua hervida de isaño, con descanso una semana y así sucesivamente por seis meses, ahora ya estoy bien" $(\mathrm{E}=6)$.

Otra de las informantes afirma: "El isaño silvestre negro es bueno para curar el mal de próstata de las personas de edad avanzada. El isaño normal puede ser consumido cocinado y también beber su agua por varios días en ayunas. El más efectivo es el isaño crudo licuado, pero es muy amargo ... mis familiares, mis abuelos, mis tíos y 
mi papá no sufren de la próstata porque siempre consumen como prevención ..., los que viven en la ciudad no consumen pue, como alimento, sino, solo quieren comer cuando ya se encuentran mal, o sea para curarse" $(E=7)$. "Antes los hombres no conocían el mal de próstata porque comían el isaño, ahora casi no comen, entonces por eso bastante gente tiene problema de próstata" $(E=3)$.

En estas poblaciones rurales el isaño es consumido como medida de prevención para evitar la inflamación de la próstata y en el caso de las mujeres para el tratamiento de los problemas renales.

\section{Plantas complementarias usadas para el tratamiento}

En la región altoandina crecen las plantas y arbustos que son utilizadas como medicina natural para curar las enfermedades de los pobladores. Para aliviar y curar los dolores del riñón y la próstata se usan como complementarias algunas de estas plantas de la sierra y de la selva. Por ejemplo, "la chachacoma (Senecio oreophyton) planta silvestre de la región altoandina; la quiswara (Buddleja coriácea Remy) planta silvestre de la región altoandina; la mullacka (Physalis Angulata) planta silvestre de la selva; el pinko pinko o cola de caballo (Equisetum); la anu chapi (Xanthium spinosum)" $(\mathrm{E}=5)$. Estas plantas, entre otras, complementan el remedio casero, pero efectivo, para tratar ciertas enfermedades como la prostatitis.

\section{Proceso de tratamiento de la próstata con isaño}

En las comunidades campesinas altoandinas hay más de un curandero que se dedica a la sanación de la prostatitis en las personas de avanzada edad y algunos jóvenes. Los entrevistados narran dichas experiencias:

"Para hacer el tratamiento a una persona mal de próstata se tiene que hacer una serie de análisis, se debe comenzar con el diagnóstico a través de los síntomas que presenta la persona, luego mediante la radiografía del cuy y también corroborando con la ecografía especializada, esto se hace con la finalidad de tratar de manera particularizada a los pacientes, recién se puede proceder con el tratamiento ... isaño "pajarillu" es para curar el mal de la próstata, no es el isaño de consumo, su característica es el tamaño largo y delgado" $(E=2)$.
El informante $(E=2)$ habría curado a varias personas enfermas de próstata de diferentes lugares del país como Lima, Arequipa, Tacna. Incluso, dijo haber llegado hasta Argentina para tratar este mal. Él no hace publicidad para posicionarse como tal, sino que los mismos clientes lo hacen, comunicándose entre ellos o recomendándolos.

Para el tratamiento efectivo explicó que el "pajarillu tiene que estar fresco para que surta el efecto curativo, porque los guardados o soleados pierden sus propiedades curativas. El tratamiento puede ser tomando licuado de isaño crudo con alguna fruta para neutralizar la acidez y, también se puede tomar el agua hervida de isaño semi cocinado. El tratamiento varía según el estado de cada enfermo, tres veces al día, pero no puede ser por más de cuatro días cada tratamiento. En el caso que se excede el tratamiento afecta a la capacidad visual del ojo" $(\mathrm{E}=2)$.

Cuando señala que cada paciente requiere un tratamiento particular, quiere decir que se necesita agregar algunas yerbas tanto de la selva como de la zona altoandina, a las que hace alusión el informante $(E=5)$. Según sea el grado de complicación del mal de la persona, puede requerirse desde dos hasta cuatro tratamientos. La evidencia del resultado del tratamiento es que las mismas personas regresan para agradecerle por haberles sanado la próstata.

Para el curandero de la comunidad de Maquercota de la provincia de El Collao, el proceso es el siguiente: "Extraer el jugo del isaño con un extractor. El jugo extraído se debe mezclar con el agua hervida de las hierbas. El mezclado del extracto de isaño con el agua de estas hierbas, se toma en forma de mate durante el día y parte de la noche, antes de dormir. Se recomienda tomar en ayunas (antes de desayunar), antes del almuerzo y antes de dormir" $(\mathrm{E}=5)$.

Las experiencias de los entrevistados demuestran que el isaño silvestre presenta mayor efectividad para el tratamiento de los problemas de próstata, principalmente cuando la persona tiene dificultades al orinar: "Hay otro medio salvaje, pajarillu se llama, es más efectivo para la próstata, para aquellas personas que ya no pueden orinar, le está doliendo para orinar, este pedacito se hace hervir en agua y lo toma dos o tres veces y ya está orinando, es efectivo, es el isaño silvestre y se llama pajarillu, acá tenemos. Muchos vienen a buscarme para curarse, y les digo que tomen pajarillu y normal están... cuando consumen isaño cultivado no tienen problemas de próstata" (E-3). De esta 
forma se evidencia que el isaño pajarillu es el más efectivo en el tratamiento de la prostatitis y el isaño de consumo previene la enfermedad.

\section{Diferencia entre isaño de consumo y pajarillu para el tratamiento}

La información obtenida revela que existen diferencias en la capacidad curativa entre el isaño de consumo y el silvestre o pajarillu.

"Pajarillu es muy bueno para todo, por ejemplo cuando tenemos que dosificar a nuestros ganados como la oveja y vaca, hay que coger 10 unidades de pajarillu, hay que hacer hervir en una olla y cuando está frío hacer tomar a los ganados esto mata a los gusanos estomacales de los animales. El pajarillu también, nos hacemos hervir para nosotros, en algunas veces los niños orinan cada rato y los adultos también para esto es bueno agua de pajarillu, el que está mal tiene que tomar agua de isaño pajarillu por un periodo de 15 días por lo menos. Mi abuelita decía que comamos siempre isaño para no enfermarnos de la próstata" $(E=5)$.

El otro informante explica que "el isaño utilizado para el consumo humano en sus diferentes clases y colores no es para curar el mal de la próstata, sino es para prever dicha enfermedad, conforme se utilizan como alimentación humana no permite inflamar la próstata" $(E=2)$.

La capacidad curativa del isaño, tanto el pajarillu como el de consumo, varía para tratar el problema de próstata. "El isaño negro de consumo, me imagino que solo tiene un $30 \%$ de efectividad en comparación con el tubérculo negro silvestre o pajarillu, por lo que no es bueno para curar el mal de la próstata" $(\mathrm{E}=2)$. Por otro lado, el informante advierte que "los animales no deben comer el pajarillu, ni las hojas, ni el tubérculo porque los mata, es muy fuerte" $(E=2)$.

El tubérculo de isaño tanto de consumo como el pajarillu es importante para las poblaciones altoandinas, porque permite prevenir y curar los problemas de salud de las personas y de sus animales, que son víctimas de ciertos parásitos que pueden afectar su crecimiento y desarrollo.

\section{Interpretación de los datos}

Rescatar los saberes ancestrales y culturales de las poblaciones altoandinas respecto al uso de las plantas de su entorno para su alimentación y la curación de ciertas enfermedades es trascendental, debido a que muchos de ellos están desapareciendo por la indiferencia de la ciencia moderna. Estos conocimientos practicados durante miles de años por las sociedades antiguas y rurales tuvieron resultados positivos en la lucha contra enfermedades desconocidas, en épocas en que no había hospitales ni médicos. Si la ciencia pudiera ponderar la importancia de dichos conocimientos, la ciencia de la salud progresaría mucho más para curar numerosas enfermedades actuales y prevenir otras en el futuro.

El isaño (Tropaeolum tuberosum Ruiz y Pavón) llamado también cubio, añu, mashua, es una de las plantas cultivadas cuyo fruto es el tubérculo para el consumo humano, la ssp. Tuberosum, y la ssp. silvestre Sparre para el medicamento, muy poco conocido (Bulacio y Ayarde, 2012). El hábitat de esta planta en la región de Puno es sobre los 3 $800 \mathrm{msnm}$, que es la altitud apropiada donde la cultivan en cantidades mínimas los pobladores rurales y donde crece el isaño silvestre en el campo abierto alrededor de los pedregales. Es el lugar frígido (Aruquipa et al., 2017). Para atender requerimientos mayores de la población no es lo suficiente, por lo que amerita pensar en estrategias que permitan producir mayor cantidad, sobre todo el silvestre para curar la prostatitis, y considerar en la alimentación cotidiana el comestible para prevenir la enfermedad.

Existen variedades de isaño en el altiplano, diferenciadas principalmente por sus colores (Inostroza et al., 2015). En el estudio se encontró el isaño de consumo en los colores amarillo zapallo, amarillo de ojos violeta, negro, blanco humo y ojos negros; y el silvestre: negro, rosado, blanco humo y amarillo. Son largos y delgados. Estos tubérculos están comprendidos dentro de las variedades mencionadas por el citado autor. Cada variedad puede ser utilizada para el consumo en diferentes presentaciones según las costumbres y usos de los pueblos: sancochado, sancochado congelado, mermelada, extracto, puré, entre otras. Mientras que el silvestre o pajarillu es considerado únicamente para la curación de la próstata y otros problemas del sistema urinario. De esta forma concuerda con Aire - Artezano et al. (2013), quienes indican que se emplea como tratamiento en litiasis renal y dolencias genitourinarias.

Según el conocimiento y los saberes de los pobladores y curanderos de la zona altoandina, el 
isaño de consumo no es para curar la prostatitis que sufren las personas de avanzada edad, sobre todo, la población urbana, debido a que casi nunca lo han considerado como parte de su alimentación. El isaño de consumo utilizado como alimentación cotidiana cumple el papel de prevención de la inflamación de la próstata de las personas vulnerables y adultos. Por ello, la población en general debería incluirlo en su plan de alimentación a fin de evitar la enfermedad de la próstata debido a su alto contenido nutracéutico.

Cada pueblo altoandino a través de sus curanderos tiene una particular forma de elaborar el medicamento a base de isaño silvestre o pajarillu para curar la próstata. Pero la información mayoritaria indica que debe ser crudo licuado para que sea efectivo o beber el agua del tubérculo semicocinado por varios días, según el caso particular de cada paciente. Sin embargo, no se debe exceder, porque las dosis más altas de este tubérculo pueden ser reversibles 24 días después de la finalización del tratamiento (Leiva-Revilla et al., 2012). Algunos entendidos en la curación realizan el diagnóstico previo al tratamiento, a fin de conocer el estado del paciente y de acuerdo al resultado obtenido agregar algunas yerbas complementarias para hacerlo más efectivo en el caso de prostatitis avanzada.

Muchas personas que adolecían del problema de la próstata se habrían curado con el isaño silvestre bajo la metodología indicada por sus curanderos. Estas personas previamente habrían acudido a un hospital a solicitar el tratamiento especializado, pero sin resultado. Los mismos pacientes curados reconocen y respetan como un gesto de agradecimiento a la persona que los sanó $\mathrm{y}$ hacen la publicidad boca a boca o recomiendan a los clientes potenciales sobre la sabiduría de su curandero. De esa forma la cultura de las poblaciones rurales altoandinas es cultivada bajo el principio de reciprocidad. Ello no solo ocurre en este caso, sino que la reciprocidad está presente en diferentes escenarios de las comunidades campesinas, sobre todo en la zona sur de la región de Puno. Así la historia de la medicina tradicional enfocada en ciencias sociales tiene lugar en las dimensiones biológica, cultural, psicológica y espiritual (Perdiguero y Comelles, 2000).

El pajarillu, además del uso para la próstata, es utilizado en otro tipo de tratamientos, por ejemplo, para desparasitar el ganado vacuno y ovino. La población altoandina aprovecha las bondades de este tubérculo para cuidar la salud de sus animales, a fin de garantizar el mejor crecimiento y alcanzar un peso apropiado en un tiempo prudente. Así el isaño pajarillu puede ser utilizado como medicina para curar la próstata en los humanos y desparasitar a los animales que crían en sus comunidades.

Como se puede ver desde la experiencia de los habitantes rurales de las comunidades altoandinas de la región de Puno, el isaño posee bondades funcionales y nutricionales para el uso de la humanidad. Estos saberes datan de tiempos ancestrales, cuando no se tenía desarrollada la ciencia de la salud. Son sapiencias milenarias de nuestros antepasados que han permitido luchar y vencer muchas enfermedades. Por eso merecen ser rescatados, revalorados, vigorizados y estudiados a profundidad para el beneficio de la sociedad. En la actualidad, solo en algunos sectores de las poblaciones altoandinas se siguen cultivando estos conocimientos, pero muchos están en proceso de desaparición junto con las personas de avanzada edad que fallecen.

\section{Conclusiones}

Según la experiencia de los pobladores altoandinos, el isaño silvestre también conocido como pajarillu es un tubérculo efectivo para curar la prostatitis, y se puede consumir crudo licuado con alguna fruta para aliviar la acidez o beber el agua hervida de isaño semicocinado por varios días, según el requerimiento de cada paciente. Además es utilizado para desparasitar a los animales criados, como el vacuno. El pajarillu es una planta silvestre que crece libremente en la región altoandina y muy poco usado como planta ornamental en algunos hogares altoandinos. Aparece en cada campaña agrícola entre los meses de diciembre, enero, febrero, marzo y abril.

El isaño de consumo en sus diferentes variedades y colores, según los conocimientos y saberes altoandinos, no curaría la prostatitis, sino que solo sería para prevenir la enfermedad, debido a que tiene bajo porcentaje de componentes nutracéuticos en comparación con el pajarillu. Las personas que viven en el medio rural consumen el isaño con regularidad, por lo que no serían propensas a sufrir este mal. En cambio aquellas que no lo utilizan para prevenir, generalmente 
tendrían problemas de próstata. La campaña agrícola de isaño se realiza anualmente, desde noviembre hasta abril, y este último mes es la etapa de la cosecha y almacenado para el consumo durante el año.

El isaño silvestre-pajarillu puede ser una solución alternativa para curar la prostatitis que sufren muchas personas, tanto los pobladores altoandinos como de otros lugares geográficos, quienes son afectados en el normal desenvolvimiento de su vida cotidiana. Por ello se requiere una mejor planificación respecto a la estrategia de domesticación de este tubérculo silvestre, que se puede cosechar en cantidades mayores para atender la demanda en la curación del problema de próstata.

\section{Agradecimientos}

A la Universidad Nacional del Altiplano de Puno por el apoyo financiero para el desarrollo de la investigación.

\section{Literatura Citada}

Aire-Artezano, G.; Charaja-Vildoso, R.; Cruz-Santiago, H.D.L.; Guillermo-Sánchez, B.; Gutarra-Vela, M.; HuamaníCharagua, P.; Chilquillo, G.J.; Nicho-Póvez, M.; Ochoa, M.; Pari, R.

2013. Efecto de Tropaeolum tuberosum frente a la hiperplasia prostática benigna inducida en ratas Holtzman. CIMEL, 18(1): 1-13.

Aruquipa, R.; Trigo, R.; Bosque, H.; Mercado, G.; Condori, J. 2017. El isaño (Tropaeolum tuberosum) un cultivo de consumo y medicina tradicional en Huatacana para el beneficio de la población boliviana. RIIARn, 3(2): 146-151.

Benítez, L.; Pagán, M.J.; Martínez-Monzó, J.; García-Segovia, P. 2016. Propiedades funcionales de tubérculos nativos de la región andina de Chimborazo (Ecuador): una revisión. Rev. Esp. Nutri. Comunitaria, 22(4): 28-33.

Bulacio, E.; Ayarde, H.

2012. Aspectos ecológicos y distribución de Tropaeolum tuberosum ssp. silvestre (Tropaeolaceae) en Argentina. Bol. Soc. Argent. Bot., 47(1-2): 97-101.

Campos, D.; Noratto, G.; Chirinos, R.; Arbizu, C.; Roca, W. \& Cisneros-Zevallos, L.

2006. Antioxidant capacity and secondary metabolites in four species of Andean tuber crops: native potato (Solanum sp.), mashua (Tropaeolum tuberosum Ruiz \& Pavón), oca (Oxalis tuberosa Molina) and ulluco (Ullucus tuberosus Caldas). J Sci Food Agric, 86: 1481-1488.

Carpio, J.; Apaza, J.; Calderón, A.; Cutipa, G.; Alcántara, A.; Velásquez, E.; Ramos, R.; Tapia, F.; De la Vega, E.; Coasaca, W.; Núñez, M.; Alanoca, V.; Chambilla, A.; Onofre, L.; Antezana, D.; Puma, J.; Tumi, E.

2016. Hojas, flores y raíces en la medicina tradicional para su validación científica. Altiplano. Puno, Perú. 224 p.

Cruz, J.; Valverde, M.E.; Ybáñez, R.

2017. Efecto del extracto acuoso de Tropaeolum tuberosum "mashua" sobre el deterioro de la memoria y lipoperoxidación inducida con fluoxetina en hígado en Rattus rattus var. albinus ooforectomizadas. Rev. Perú Med Integrativa, 2(2): 19-25.

Espinoza, E.S.; Monteghirfo, G.M.

1999. Determinación de la composición de aminoácidos de mashua (Tropaeolum tuberosum) mediante cromatografía líquida de alta performance (HPLC). Boletín de la Sociedad Química del Perú, LXV, 216-225.
Guevara-Freire, D.; Valle-Velástegui, L.; Barros-Rodríguez, M.; Vásquez, C.; Zurita-Vásquez, H.; Dobronski-Arcos, J.; Pomboza-Tamaquiza, P.

2018. Nutritional cmpositions y bioactive components of mashua Tropaeolum tuberosum Ruiz y Pavón)]. Tropical and Subtropical Agroecosystems, 21: 53-68.

Inostroza, L.A.; Castro, A.J.; Hernández, E.M.; Carhuapoma, M.; Yuli, R.A.; Collado, A.; Córdova, J.

2015. Actividad antioxidante de Tropaeolum tuberosum Ruiz \& Pavón (mashua) y su aplicación como colorante para yogur. Ciencia e Investigación, 18(2): 83-89.

Leiva-Revilla, J.; Cárdenas-Valencia, I.; Rubio, J.; GuerraCastañón, F.; Olcese-Mori, P.; Gasco, M.; Gonzales, G.F. 2012. Evaluation of different doses of mashua (Tropaeolum tuberosum Ruiz y Pavón) on the reduction of sperm production, motility and morphology in adult male rats. Andrología, 44: 205-212.

Malice, M.; Bizoux, J.P.; Blas, R.; Baudoin, J.P. 2010. Genetic Diversity of Andean Tuber Crop Species in the in situ Microcenter of Huanuco, Peru. Crop Science, 50: 1915-1923.

Morillo, A.C.; Morillo, Y.; Tovar, Y.P.

2016. Caracterización molecular de cubios (Tropaeolum tuberosum Ruiz y Pavón) en el departamento de Boyacá. Rev. Cienc. Agr., 33(2): 32-42.

Perdiguero, E.; Comelles, J.M.

2000. Medicina y cultura: Estudios entre la antropología y la medicina. Ediciones Bellaterra. Barcelona, España. 448 p.

Rodríguez, G.; Gil, J.; García, E.

1999. Metodología de la investigación cualitativa. Aljibe. Málaga, España.

Salluca, T.G.; Peñarrieta, J.M.; Alvarado, J.A.; Bergenståhl, B. 2008. Determination of total phenolic compounds content and the antioxidant capacity of andean tubers and roots (isaño, oca, ulluco and arracacha). Revista boliviana de química, 25(1): 58-61.

Valcárcel-Yamani, B.; Rondán-Sanabria, G.G.; Finardi-Filho, F. 2013. The physical, chemical and functional characterization of starches from Andean tubers: Oca (Oxalis tuberosa Molina), olluco (Ullucus tuberosus Caldas) and mashua (Tropaeolum tuberosum Ruiz \& Pavón). Brazilian Journal of Pharmaceutical Sciences, 49(3): 453-464.

Valle-Parra, M.; Pomboza-Tamaquiza, P.; Buenaño-Sánchez, M.; Guevara-Freire, D.; Chasi-Vizuete, P.; Vásquez, C.; PérezSalinas, M. 
2018. Morphology, phenology, nutrients and yield of six accessions of Tropaeolum tuberosum ruiz y pav (mashua) Tropical and Subtropical Agroecosystems, 21: 131-139. Vasilachis, I.

1992. Métodos Cualitativos I. Los problemas teóricoepistemológicos. Centro Editor de América Latina. Buenos Aires, Argentina. $71 \mathrm{p}$

Vásquez, J.H.; González, J.M.; Pino, J.L.

2012. Decrease in spermatic parameters of mice treated with hydroalcoholic extract Tropaeolum tuberosum "mashua". Rev. peru. biol., 19(1): 089-093.
Velásquez-Barreto, F.F.; Velezmoro, C.

2018. Propiedades reológicas y viscoelásticas de almidones de tubérculos andinos. Scientia Agropecuaria, 9(2): 189-197.

Velásquez, H.L.; Carpio, J.B.; Reyes, F.R.; Flores, D. 2013. Antropología médica: hojas, flores y raíces en el contexto sociocultural aymara del altiplano peruano. Comuni@cción: Revista de Investigación en Comunicación y desarrollo. 4(2): 53-61. 